\title{
Optical gas sensor fabrication based on porphyrin-anchored electrostatic self-assembly onto tapered optical fibres
}

\author{
Renata Jarzebinska ${ }^{1}$, Sergiy Korposh ${ }^{2}$, Stephen James ${ }^{1}$, William Batty ${ }^{1}$, Ralph Tatam ${ }^{1}$, and \\ Seung-Woo Lee ${ }^{2 \dagger}$ \\ ${ }^{1}$ School of Engineering, Cranfield University, Cranfield, Bedford MK43 OAL, UK \\ ${ }^{2}$ Department of Chemical Processes and Environments, Graduate School of Environmental \\ Engineering, the University of Kitakyushu, 1-1 Hibikino, Wakamatsu-ku, Kitakyushu 808-0135, \\ Japan
}

(Corresponding author*: Seung-Woo Lee; E-mail: leesw@kitakyu-u.ac.jp) 


\begin{abstract}
Tapered optical fibres with nano-assembled coatings of thicknesses of order tens of nanometres were used for the detection of ammonia gas. The film coating was composed of alternate layers of tetrakis-(4-sulfophenyl) porphine (TSPP) and poly(allylamine hydrochloride) (PAH), which were deposited using the electrostatic self-assembly process (ESA). Exposure of a PAH/TSPP nano-assembled non-adiabatic tapered optical fibre with a waist diameter of $10 \mu \mathrm{m}$ to ammonia induced significant optical changes in the transmission spectrum of the optical fibre. The fibre optic sensor showed a linear sensitivity to the concentration of ammonia in the range of 10-100 ppm, with response and recovery times less than 100 and $240 \mathrm{sec}$, respectively. The $3 \sigma$ limit of detection (LOD) was estimated to be ca. 2 ppm.
\end{abstract}

Keywords: tapered optical fibre gas sensors; electrostatic self-assembly process; porphyrin; ammonia gas 


\section{Introduction}

Optical techniques offer a powerful tool for the characterization of chemical and biological systems (Rees 2002, Del Villar 2005, Grattan 1999). In particular, the coating of optical waveguides with nanomaterials that exhibit changes in optical properties upon exposure to targeted chemical species offers a prospect for the development of sensitive optical devices based upon the interaction of the evanescent field of the optical waveguide with the coating layer (Cusano 2005, James 2006, Gu 2007). For instance, different sensitive materials, such as bromocresol purple (Cao 2005), oxazine 170 perchlorate (Tao 2007) and fluorescence agents (Kara 1993) have been used for the development of fibre-optic ammonia gas sensors. Among the optical waveguide devices that have been investigated, tapered optical fibre sensors are able to measure environmental parameters (refractive index, chemical concentration, etc.) with high sensitivity owing to the large proportion of the energy of the propagating mode extending into the surrounding environment in the form of an evanescent field (Corres 2007, Leung 2007, James 2006).

A tapered optical fibre may be fabricated by simultaneously heating and stretching a short section of a single mode optical fibre. This creates a region of fibre with reduced and uniform diameter (the waist) that is bounded by conical sections where the diameter of the fibre changes to merge the tapered section with the unperturbed surrounding single mode fibre. The optical properties of the tapered fibre waveguide are influenced by the profile of the conical tapering sections, by the diameter of the taper waist and by the optical thickness of the surrounding medium. The proportion of the power in the evanescent field, and thus the interaction with the surrounding medium, increases with decreasing the diameter of the taper waist (Jarzebinska 2009). In the tapering section, the guided mode of the single mode fibre is converted into a mode of the waist. In adiabatic tapers this is achieved without coupling to higher order modes. In non-adiabatic tapers the taper profile is such that a proportion of the light is coupled into 
higher order modes of the tapered section, which interfere to produce the channeled spectra reported for tapers of diameter of order $5 \mu \mathrm{m}$ (Jarzebinska 2009, Corres 2008).

The tapered area of the optical fibre facilitates evanescent wave spectroscopy, in which the absorption spectrum of the surrounding medium is measured. Alternatively, the influence of the surrounding medium on the properties of the optical modes of the tapered waveguide can be explained as a change in the refractive index, i.e. it will operate as a refractometer.

Different immobilization procedures based on covalent and noncovalent bonds have been used to facilitate the deposition of functional films onto optical fibres (James 2006). The electrostatic self-assembly (ESA) method is a useful candidate for the preparation of nano-assembled films of molecular levels on a variety of surfaces. This deposition technique is still expanding its potential because of its versatility in the fabrication of ordered multilayer films and the ability to deposit both inorganic and organic materials (Ariga 1997, Lee 1998).

In previous work it was demonstrated that porphyrin compounds can be used as functional coating materials for optical sensors because their optical properties (absorbance and fluorescence features) are influenced by chemicals to which they are exposed (Korposh 2006, Korposh 2009, Leung 2007). In general, changes of the optical properties of porphyrin are induced by (i) solvent effects, (ii) redox reactions, (iii) protonation or metallation of core nitrogen atoms, (iv) electron-electron interaction, (v) electronic changes due to structural changes such as flattening and distortion, and (vi) interactions between porphyrins (aggregation) (Takagi 2006). In particular, tetrakis-(4-sulfophenyl) porphine (TSPP) can form aggregates with distinctive optical features that correspond to certain type of aggregation (Korposh 2006). These aggregated structures can be altered by the presence of the particular gas that induces protonation/deprotonation of the pyrolle ring with concomitant changes in optical spectrum. This can be employed for the gas sensor development (Korposh 2006, Korposh 2009).

In this study, the previous findings were extended further and the ESA method have been used to deposit a multilayer porphyrin film over the tapered region of a single mode optical fibre with 
the aim of demonstrating a gas sensor. The effect of the polycation on the optical properties and structure of the multilayer porphyrin film was thoroughly studied. It is suggested that by using poly(allylamine hydrochloride), $\mathrm{PAH}$, for the porphyrin film preparation instead of poly(diallyldimethylammonium chloride), PDDA, the form of the aggregation of the TSPP are modified and provide improved optical properties that facilitate the detection of wider class of chemicals. Moreover, for the first time, to the best of our knowledge, the analyte-induced refractive index change of the prepared multilayer porphyrin film was monitored using tapered optical fibres. In addition, the effect of the taper waist diameter on the sensor performance was studied by fabrication of tapers with waist diameters of 9,10 and $12 \mu \mathrm{m}$.

\section{Experimental}

\section{Reagents and chemicals}

Porhyrin tetrakis-(4-sulfophenyl)porphine (TSPP, $\left.M_{\mathrm{w}}=934.99\right)$, sodium hydroxide $(\mathrm{NaOH})$ and potassium hydroxide $(\mathrm{KOH})$ were purchased from Tokyo Kasei, Japan. Poly(allylamine hydrochloride) (PAH, $\left.M_{\mathrm{r}}: 56000\right)$ was obtained from Sigma-Aldrich. All of these chemicals were guaranteed reagents, and used without further purification. Standard ammonia gas of 100 ppm in dry air was purchased in cylinder from Japan Air Gases Corp. Deionized pure water (18.3 M $\Omega \cdot \mathrm{cm})$ was obtained by reverse osmosis followed by ion exchange and filtration (Millipore, Direct-QTM). A single mode silica optical fibre (Fibercore SM750 with cut-off wavelength $635 \mathrm{~nm}$, core diameter of $8 \mu \mathrm{m}$ and cladding diameter of $125 \mu \mathrm{m}$ ) was used in these experiments.

\section{Preparation of films}

The detailed description of the fibre tapering procedure can be found elsewhere (Jarzebinska 2009). Briefly, a single mode silica optical fibre was tapered using the heat and pull technique. 
Firstly, the polymer buffer coating was removed from a $50 \mathrm{~mm}$ long section in the middle of a $\sim 1 \mathrm{~m}$ length of the optical fibre using a mechanical stripper. The stripped section of the optical fibre was then fixed on a 3-axis flexure stage (NanoMax ${ }^{\mathrm{TM}}$, Thorlabs) and exposed to the flame produced by a gas burner (max temperature $1800^{\circ} \mathrm{C}$ ) for approximately $60 \mathrm{sec}$ while the ends of the fibre were pulled in opposite directions using translation stages. Nonadiabatic optical fibre tapers of diameters 9,10 and $12 \mu \mathrm{m}$, all having a taper waist of $20 \mathrm{~mm}$, were fabricated. The dimensions of the tapers were determined using a digital optical microscope, DZ3 Union Optical Co., Ltd., Japan.

An electrostatic layer-by-layer (LbL) method was employed for the deposition of multilayer porphyrin sensor films onto the tapered optical fibres, as described elsewhere (Korposh 2009). Briefly, the tapered region of the optical fibre was fixed in a hand-made Teflon holder, rinsed with deionised water and exposed to $1 \mathrm{wt} \%$ ethanolic $\mathrm{KOH}$ for $20 \mathrm{~min}$, leading to a negatively charged surface. The optical fibre was then exposed to a solution containing a positively charged polymer poly(allylamine hydrochloride) (PAH) for $20 \mathrm{~min}$, so that a monolayer of PAH was deposited onto the surface of the fibre. The $\mathrm{pH}$ of the PAH solution was adjusted to 10.5-11 using $\mathrm{NaOH}$. Subsequently, the tapered optical fibre was rinsed with distilled water and dried by flushing with nitrogen gas. The fibre was then exposed to a solution of a functional porphyrin dye, tetrakis-(4-sulfophenyl) porphine (TSPP) for $20 \mathrm{~min}$, which provides the sensing capability. The fibre was again rinsed with distilled water and dried. The multilayer film was built up by alternating exposure to the PAH and TSPP solutions and stopped when a five bilayer PAH/TSPP film was prepared, giving a thickness of ca. $10 \mathrm{~nm}$ (Korposh 2006). This procedure was applied to the optical fibres with different tapered diameters of 9,10 and $12 \mu \mathrm{m}$. Additionally, in order to check the film properties, the PAH/TSPP film was also assembled onto a quartz substrate using the same deposition procedure.

\section{Optical measurements}


Absorption spectra of the PAH/TSPP film deposited on quartz substrates were measured using a double beam Jasco V-550 UV-vis spectrophotometer. The transmission spectra of the optical fibre tapers were characterized by coupling the output from a tungsten-halogen light source (HL-2000, Ocean Optics) into the proximal end of the optical fibre, and coupling the output from the distal end into a spectrometer (HR2000, Ocean Optics). This system was used to record transmission spectra of the tapered fibre during film deposition and during its subsequent exposure to ammonia.

For ammonia gas measurements the desired concentration was produced using a two-arm flow system, as described elsewhere (Korposh 2006, Korposh 2009).

For humidity measurements, a humidity logger (Hygrochron, KN Laboratories: relative humidity range of $0-95 \%$; accuracy $\pm 5 \%$ at $25{ }^{\circ} \mathrm{C}$ in the range of $20-80 \% \mathrm{RH}$ and reading resolution $0.1 \%$ ) was used to measure humidity inside the measurement chamber.

\section{Results and Discussion}

\section{Optical spectra of the PAH/TSPP film}

Figs. 1a and $1 \mathrm{~b}$ show the evolution of the absorption spectra measured during the deposition of a 5-bilayer PAH/TSPP film, (PAH/TSPP) $)_{5}$, onto a quartz substrate with PAH (Fig. 1a) and TSPP (Fig. 1b) outermost layers, respectively. The largest change in absorbance due to deposition of the (PAH/TSPP) bilayers was observed at $420 \mathrm{~nm}$, which corresponds to the Soret band of TSPP. In accord with our previous reports of the deposition of poly(diallyldimethylammonium chloride)/TSPP (PDDA/TSPP) alternate layers (Korposh 2006, Korposh 2009), the UV-vis absorbance increases with the number of the TSPP deposition cycles (inset in Fig. 1b), indicating a uniform film growth on the quartz substrate. In contrast to the PDDA/TSPP film, however, the $1^{\text {st }}$ Soret-band $(420 \mathrm{~nm})$ is more pronounced in the PAH/TSPP film and is accompanied by the weakly developed $2^{\text {nd }}$ Soret-band $(490 \mathrm{~nm})$ and 
Q-bands (500-700 nm), suggesting that monomeric structures of TSPP can be preferably formed (Korposh 2006, Korposh 2009). It is possible that the amine groups of PAH induce the partial deprotonation of TSPP and reduce the amount of $J$-aggregates of TSPP. With a PAH outer layer, a strong absorbance peak at $420 \mathrm{~nm}$ (protonated TSPP) along with the weak band at $410 \mathrm{~nm}$ (non-protonated TSPP) and a number of small Q-bands (500-700 nm) were observed, indicating the desorption of the TSPP $J$-aggregates. However, the absolute amount of the adsorbed TSPP molecules is lower than that observed in the PDDA/TSPP film because of weak cationic property of PAH. Consequently, films with a TSPP outermost layer will be more sensitive to exposure to analytes such as ammonia than those with a PAH outermost layer. On the other hand, the configurations of TSPP inside the film are more diverse in the PAH/TSPP film (Selyanchyn 2011) as compared to those in the PDDA/TSPP film, in which TSPP exists as a zwitterionic form and the $J$-aggregation arrangement is predominant (Korposh 2006, Korposh 2009). In particular, as inferred from absorption spectra, in the PAH/TSPP film TSPP exists in monomeric protonated (peaks at 420 and $658 \mathrm{~nm}$ ) and non-protonated $(410 \mathrm{~nm})$ states and the protonated zwitterionic form is required for creation of the $J$-aggregates (peaks at 490 and 706nm). The presence of TSPP in different states provides unique optical properties and rich absorption spectra that can be utilised for the detection of different chemical compounds.

\section{Properties of the tapered fibres}

Figs. $2 \mathrm{a}-2 \mathrm{c}$ compare the transmission spectra of the tapered fibres with 9,10 and $12 \mu \mathrm{m}$ waist diameters, respectively, measured in air and water. In general, the transmission spectrum of a tapered optical fibre depends strongly on the geometry of the taper; i.e. waist diameter, length of the taper region and taper angle. If the taper angle is small enough to ensure negligible transfer of power from the fundamental core mode to higher order modes of the taper waist, then the taper is termed adiabatic. In non-adiabatic tapers, the fundamental mode couples to higher order 
modes of the taper waist. Interference, or beating, between the mode results in oscillations in the transmission spectrum. For smaller taper diameters it is possible to observe the excitation of two hybrid modes, $\mathrm{HE}_{11}$ and $\mathrm{HE}_{12}$, of the tapered waist. These two modes propagate, with different effective refractive indices, to the end of the tapered waist, and then will couple back to the $\mathrm{LP}_{01}$ mode of the fibre through the second conical region. Interference between these modes produces a sinusoidal channelled spectrum (Black 1991, Kieu 2006). This effect has been used previously to demonstrate sensors for temperature and surrounding refractive index, based upon the differential response of the two modes of the tapered region (Kieu 2006). As the taper diameter increases the number of excited modes increase, resulting in more complex spectra. The form of the spectra shown here indicates clearly that the tapers are non-adiabatic.

When the tapered fibre is immersed into water, the wavelength of the channelled spectra undergoes a red shift along with a change in the amplitude of the optical spectrum due to increase of the refractive index (RI) of the surrounding medium from 1.00 (air) to 1.33 (water). The wavelength shift of the channeled spectrum is a result of a differential change in the propagation constants of the modes within the tapered region (Corres 2007, Jarzebinska 2009), while the reduction in amplitude is a result of the reduced confinement of the modes that accompanies immersion in water, which has a higher refractive index than that of air. Interestingly, the wavelength shifts (determined as an average value across the spectrum) of the spectral features are 28,16 and $15 \mathrm{~nm}$ for the 9,10 and $12 \mu \mathrm{m}$ tapered optical fibres, respectively, suggesting that fibre with thinner waist diameter possesses higher sensitivity to RI change.

\section{Film assembly onto the tapered fibres}

In this study, the changes in the transmission spectrum during the film deposition is influenced by two effects; the absorption spectrum of TSPP and differential changes in the propagation 
constants of the modes of the tapered fibre section. The response of the transmission spectrum to increasing numbers of deposited layers is shown in Fig. 3. The channeled spectra undergo a red-shift due to the differential changes in the effective refractive index of the $\mathrm{HE}_{11}$ and $\mathrm{HE}_{12}$ modes of the tapered waist in response to the increasing optical thickness of the coating, for all tapered fibres. There is also a strong decrease in the measured power at $700 \mathrm{~nm}$ when the tapered optical fibre is immersed into the TSPP solution (Fig. 3d) (Takagi 2006).

The transmission of the tapered fibre measured near $700 \mathrm{~nm}$ decreases with increasing number of deposited bilayer. It is interesting to note that, at stages during the coating deposition when the structure of the coating is such that TSPP outermost layer, the transmission of the tapered fibre at $700 \mathrm{~nm}$ measured in solution and in air (Figs. 3a and 3b, respectively) is lower than when PAH is the outer layer (Figs. 3c and 3d). At the same time the wavelength shift of the channeled spectra was largely independent of which material formed the outer layer (Figs. 3b and 3c). These results indicate that tapered fibres respond not only to the film thickness and RI change, but are also sensitive to the absorbance of the deposited TSPP sensing layer. Moreover, the red shift of the transmission peaks correlates with the number of deposited layers, indicating the uniform deposition of the TSPP and PAH layers on the tapered optical fibres. When the PAH is deposited as an outermost layer, the wavelength shifts are approximately 1.0, 1.2 and $1.3 \mathrm{~nm}$ per bilayer for the 9,10 , and $12 \mu \mathrm{m}$ diameter tapered fibres modified with (PAH/TSPP) ${ }_{5}$ film, respectively (data for 9 and 12 um diameters not shown in figures). On the other hand, when TSPP formed the top layer, the wavelength shifts are 4.2, 1.1 and $1.4 \mathrm{~nm}$ per bilayer for the 9, 10, and $12 \mu \mathrm{m}$ diameter tapered fibres (Fig. 4), respectively, indicating that tapered fibre with the $9 \mu \mathrm{m}$ waist diameter is more sensitive to changes in RI of the coating than are the fibres with 10 and $12 \mu \mathrm{m}$ waist diameters. It should be noted that these results are in accord with data obtained when fibre was immersed into water. Moreover, these data correlate well with the linear dependence of the absorbance on the number of the cycles of the PAH/TSPP film that were deposited onto a quartz substrate (Fig. 1b). 
Most plausibly, the RI of the film is higher when TSPP is deposited as an outer layer due to $J$-aggregates, which is destroyed when PAH is an outer layer. In addition, TSPP is partly removed from the PAH/TSPP film when exposed to PAH solution as inferred from absorption spectra (Figs. 1a and 1b). In other words the PAH/TSPP film is denser when TSPP is an outer layer leading to higher RI value of the film as compared with PAH.

The dynamic response of the transmission of the tapered fibre measured at $700 \mathrm{~nm}$ (inset of Fig. 3a) for the deposition of each TSPP layer saturated within $100 \mathrm{sec}$, indicating that the deposition of the TSPP layer is complete within $100 \mathrm{sec}$. A reliable deposition time for the PAH layer, however, could not be obtained due to limitations of the measurement system. Consequently, to ensure the complete layer formation for both TSPP and PAH, a 20 min deposition time was employed, which was previously found to be optimal (Ariga 1997) for uniform film deposition.

\section{Response to ammonia}

A purpose designed measurement chamber was used in order to characterise the sensor performance. The tapered section of the optical fibre coated with the functional film was inserted into the chamber. The desired gas concentrations were produced using a two-arm flow system described elsewhere (Korposh 2009). The dry compressed air that was used as the carrier gas and ammonia gas of $100 \mathrm{ppm}$ concentration were passed separately through two flowmeters; the two flows were then combined to produce the desired ammonia concentration in the measurement chamber. The concentration could be controlled by adjusting the flow rates of the ammonia and the air.

The transmission spectrum was recorded with a $1 \mathrm{~Hz}$ update rate (measurement time equivalent to $1 \mathrm{sec}$ ), as the device was exposed to a given ammonia concentration and subsequently flushed with dry air. The difference spectrum was plotted by subtracting a spectrum measured at a given ammonia concentration from the spectrum recorded in the presence of dry air. The baseline spectrum and sensor response of each experiment were recorded by passing dry air through the 
measurement chamber until the signal measured at $700 \mathrm{~nm}$ reached equilibrium.

The results are shown in Fig. 5a-5d). As the ammonia concentration increased from 10 ppm up to $100 \mathrm{ppm}$, the intensity measured at $700 \mathrm{~nm}$ increased for the $10 \mu \mathrm{m}$ and $12 \mu \mathrm{m}$ diameter optical fibre tapers (Figs. 5b). Interaction of the ammonia molecule with TSPP leads to the deprotonation of the pyrolle ring of TSPP and hence influences the electrostatic interaction between the TSPP moieties in the PAH/TSPP film (Korposh 2006, Korposh 2009). Consequently, the biggest change in absorbance is observed at $700 \mathrm{~nm}$ ( $\mathrm{Q}$ band), which may be closely related to the aggregation state of the TSPP molecules (Takagi 2006).

Interestingly, when measurements were conducted using the tapered fibres with 10 and $12 \mu \mathrm{m}$ waist diameters, the channeled spectra did not exhibit a wavelength shift in response to exposure to ammonia suggesting that ammonia-induced RI change can not be measured with tapers of these diameters, possibly because the modes are tightly bound and the influence of the modes' evanescent field interaction with the coatings do not induce significant differential changes in the propagation constants (Fig. 5b). When the $9 \mu \mathrm{m}$ diameter tapered fibre coated with the (PAH/TSPP $)_{5}$ film was exposed to ammonia, a red-shift of the spectral features at 1000 and $1040 \mathrm{~nm}$ was observed that saturates with the increase of the concentration (Fig. 5c). We can assume that the wavelength red-shift of the spectral features is caused by the ammonia-induced change in the RI of the PAH/TSPP film. It should be noted that this change is not continuous and saturation occurs between 0 and $50 \mathrm{ppm}$ (Fig. 5c). As was revealed from results shown in Fig. 2, $9 \mu \mathrm{m}$ tapered fibre possesses higher sensitivity to RI change as compared to 10 and $12 \mu \mathrm{m}$ tapered fibres. The absence of the intensity change at $700 \mathrm{~nm}$ can be explained by the transmission spectrum of the $9 \mu \mathrm{m}$ tapered fibre obtained after deposition of the 5th bilayer of the PAH/TSPP film (data not shown); the optical power at $700 \mathrm{~nm}$ transmitted to spectrometer is very low and therefore it is virtually impossible to measure the small ammonia-induced intensity change. We can conclude from these results that the wavelength shift near $1000 \mu \mathrm{m}$ of the $9 \mu \mathrm{m}$ tapered fibre is sensitive to ammonia-induced RI changes of the 
coating and the change is transmitted power near $700 \mathrm{~nm}$ of the 10 and $12 \mu \mathrm{m}$ tapered fibres can be used to monitor ammonia gas concentration.

Dynamic ammonia-induced changes of the tapered fibres with 10 and $12 \mu \mathrm{m}$ waist diameters coated with the $(\mathrm{PAH} / \mathrm{TSPP})_{5}$ film were monitored at $700 \mathrm{~nm}$, as shown in Fig. $5 \mathrm{~d}$. The measurement principle for these waist diameters is based on the evanescent wave spectroscopy. The response time and recovery time $\left(t_{90}\right)$ of the sensor to increasing ammonia concentration were within $100 \mathrm{sec}$ and $240 \mathrm{sec}$, respectively. The sensitivity of the device derived from the slope of the calibration curve is $0.440 \pm 0.002 \mathrm{mV} / \mathrm{ppm}$ and estimated limit of detection (LOD) calculated using the $3 \sigma$ method is $2 \pm 0.3 \mathrm{ppm}$ (inset of Fig. $5 \mathrm{~d}$ ). It should be noted that sensitivity of the proposed sensor is ca. 3 times higher as compared to the PDDA/TSPP film assembled onto the quartz substrate (Korposh 2006). This most plausibly is owing to the higher localized energy at the taper of the optical fibre and increased interaction efficiency between the probe light and the functional film. On the other hand, the sensitivity of the fabricated device was ca. 6 times lower as compared to multimode optical fibre modified with the PDDA/TSPP film (Korposh 2009). This can be attributed to the presence of TSPP in $J$-aggregated form in higher concentration in the PDDA/TSPP film as compared to the PAH/TSPP film used in this study. However, the presence of TSPP in different forms inside the PAH film may allow to the coating to exhibit sensitivity to different chemical compounds, thus increasing the application range of the proposed sensor. This hypothesis will be thoroughly explored in the future work. In addition, the taper fibre may operate as both an evanescent wave spectroscope and as a refractometer. Thus, in contrast to solely evanescent wave spectroscopy, materials without absorbance features in the Uv-Vis range may be employed as sensitive layers, extending the utility of the chemical fibre optic sensors and class of the detectable analytes.

The fabricated device was exposed to varying relative humidity to study its effect on the sensor response. When $\mathrm{rH}$ was reduced from $70 \%$ to $10 \%$ and increased back to $70 \%$, no significant change in the transmission spectra was observed (Figs. 6a and $6 \mathrm{~b}$ ) revealing selectivity of the 
sensor to ammonia over $\mathrm{rH}$. The immunity of the sensor to $\mathrm{rH}$ change is very important for real-world practical applications where humidity is one of the major interfering parameters. For example, ammonia detection in breath is highly important non-invasive diagnostic tool in medicine (Turner 2006), but highly challenging due to high humidity present in breath. Up-to date, to the best of our knowledge, there is no sensor with satisfactory sensitivity and selectivity for the detection of ammonia in breath. In our future study of the use of this sensor application for ammonia breath measurement, the cross-sensitivity to other gases will be undertaken.

\section{Conclusions}

In summary, tapered optical fibres coated with nano-scale alternating layers of PAH/TSPP deposited using an electrostatic self-assembly approach were tested as ammonia gas sensors. The response of the transmission spectrum to the deposition of the PAH/TSPP alternate film was studied. A wavelength shift of the channelled spectra features and a decrease in transmitted optical power was observed in response to the film thickness increase and to the absorbance changes, respectively. In the case of a $9 \mu \mathrm{m}$ tapered fibres waist diameter, the red shift of the transmission peaks near $1000 \mathrm{~nm}$ is sensitive to ammonia-induced RI changes of the coating. Tapered fibres with waist diameter of 10-12 $\mu \mathrm{m}$ showed reversible and linear response to the ammonia gas with short response time and recovery time (100 and $240 \mathrm{sec}$, respectively) and limit of detection ca. $2 \mathrm{ppm}$. The sensing mechanism is based on the interaction between the ammonia and the porphyrin compound that leads to the deprotonation of the TSPP pyrolle ring and the formation of ammonium ions and is mainly accompanied by changed in transmitted power occurring at around $700 \mathrm{~nm}$ (Korposh 2009). The future challenge is to test the selectivity of the device by exposing it to different gas species. When comparing the sensing performance of the tapered optical fibres used in this study we can conclude that fibre with the smaller diameter $(9 \mu \mathrm{m})$ will be suitable for measurement of the RI change of the coating. The 
fibres with larger diameters (10 and $12 \mu \mathrm{m})$ can be employed for evanescent wave spectroscopy.

\section{Acknowledgements}

This work was supported by the MEXT via the 2nd Kitakyushu Knowledge-based Cluster Project. The authors from Cranfield are grateful to the Engineering and Physical Sciences Research Council, EPSRC, UK for funding under grants EP/D506654/1 and GR/T09149/01.

\section{References}

Ariga, K. Lvov, Y. and T. Kunitake. 1997. Assembling Alternate Dye-Polyion Molecular Films by Electrostatic Layer-by-Layer Adsorption. J. Am. Chem. Soc. 1997, 119, 2224.

Black, R, Bures, J. and J. Lapierre. 1991. Finite-cladding fibres: HE12 and local-normal-mode coupling evolution. IEE Proc. J. 138: 330.

Cao, W. and Y. Duan. 2005. Optical fiber-based evanescent ammonia sensor. Sensors and Actuators B. 110: 252-259.

Corres, J., Arregui, F. and I. Matías. 2007. Sensitivity optimization of tapered optical fiber humidity sensors by means of tuning the thickness of nanostructured sensitive coatings. Sens. and Act. B. 122: 424.

Corres, J.M., Matias, I.R., Bravo, J. and F.J. Arregui. 2008. Tapered optical fiber biosensor for the detection of anti-gliadin antibodies. Sen. and Act.B. 135: 166.

Cusano, A., Pilla, P., Contessa, L., Iadicicco, A., Campopiano, S., Cutolo, A., Giordano, M. 
and G. Guerra. 2005. Highsensitivity optical chemosensor based on coated long-period gratings for sub-ppm chemical detection in water. Appl. Phys. Lett. 87: Art. No. 234105.

Del Villar, I., Achaerandio, M., Matias, I. and F. Arregui. 2005. Deposition of overlays by electrostatic self-assembly in long-period fiber gratings. Opt. Lett. 30: 720.

Grattan, K.and B. Meggitt. 1999. Chemical and environmental sensing, Boston, USA: Kluwer Academic Publisher.

Gu, Z. and Y. Xu. 2007. Design optimization of a long-period fiber grating with sol-gel coating for a gas sensor. Meas. Sci. Technol. 18: 3530.

James, S. and R. Tatam. 2006. Fibre Optic Sensors with Nano-Structured Coatings. J. Opt. A: Pure Appl. Opt. 8: S430.

Jarzebinska, R., Cheung, C. S., James, S.W. and R. P. Tatam. 2009. Response of the transmission spectrum of tapered optical fibres to the deposition of a nanostructured coating. Meas. Sci. Technol. 20: 034001 (6pp).

Kara, S. and A. Mark. 1993. Arnold Air-gap fiber-optic ammonia gas sensor. Talanta. 40: 757. Kieu, K. and M. Mansuripur. 2006. Biconical fiber taper sensors. IEEE Photonic.Tech.Lett. 18: 2239.

Korposh, S., Kodaira, S., Batty, W., James, S. and S.W. Lee. 2009. Nano-assembled thin film gas sensor. II. An intrinsic high sensitive fibre optic sensor for ammonia detection Sensor. Mater. 21: 179 . 
Korposh, S., Takahara, N., Ramsden, J. J., Lee, S.-W. and T. Kunitake. 2006. Nano-assembled thin film gas sensors. I. Ammonia detection by a porphyrin-based multilayer film. J. of Biolog. Phys. and Chem., 6: 125.

Lee, S.-W., Ichinose, I. and T. Kunitake. 1998. Molecular Imprinting of Azobenzene Carboxylic Acid on a TiO2 Ultrathin Film by the Surface Sol-Gel Process. Langmuir. 14: 2857.

Leung, A., Shankar, P. and R. Mutharasan. 2007. Real-time monitoring of bovine serum albumin at femtogram $/ \mathrm{mL}$ levels on antibody-immobilized tapered fibers. Sen. and Act.B.123: 888.

Rees, N., James, S. and R. Tatam. 2002. Optical fiber long-period gratings with Langmuir-Blodgett thin-film overlays. Opt. Lett. 27: 686.

Selyanchyn, R., Korposh, S., Yasukochi, W. and S.-W. LEE. 2011. A preliminary test for skin gas assessment using a porphyrin based evanescent wave optical fiber sensor. Sensors \& Transducers Journal. 125: 54-67.

Takagi, S., Eguchi, M., Tryk, D. and H. Inoue. 2006. Porphyrin photochemistry in inorganic/organic hybrid materials: Clays, layered semiconductors, nanotubes, and mesoporous materials. J. of Photoch. Photob. C. 7: 104.

Tao, S., Gong, S., Fanguy, J. C. and X. Hu. 2007. The application of a light guiding flexible tubular waveguide in evanescent wave absorption optical sensing. Sensors and Actuators B. 120: 724-731.

Turner, C., Španěl, P. and D. Smith. 2006. A longitudinal study of ammonia, acetone and 
propanol in the exhaled breath of 30 subjects using selected ion flow tube mass spectrometry, SIFT-MS. Physiol. Meas. 27: 321. 


\section{Figure Captions}

Fig. 1. Evolution of the absorption spectra during deposition of a 5-cycle PAH/TSPP alternate film onto a quartz substrate measured with an outermost layer of (a) PAH and (b) TSPP. Arrows indicate the number of bilayers deposited and the inset of Fig. $1 \mathrm{~b}$ shows peak absorbance vs. the number of deposited layers measured near $410 \mathrm{~nm}$ (squares) and $706 \mathrm{~nm}$ (circles).

Fig. 2. Comparison of the transmission spectra measured in air and water using tapered optical fibres with a taper diameter of (a) $9 \mu \mathrm{m}$, (b) $10 \mu \mathrm{m}$, and (c) $12 \mu \mathrm{m}$. (d) optical images of the tapered region of the optical fibres with different waist diameter.

Fig. 3. Evolution of the transmission spectra of the $10 \mu \mathrm{m}$ tapered optical fibre during deposition of a five-cycle PAH/TSPP alternate film (a) Measured in TSPP solution and measured after drying the optical fibre with an outermost layer of (b) TSPP and (c) PAH. (d) Peak intensity measured near $700 \mathrm{~nm}$ plotted vs. the number of PAH/TSPP deposition cycles for TSPP and PAH outermost layers in water and air. Horizontal arrows indicate the number of layers deposited and the direction in which the transmission peaks are shifted. The downward arrows indicate the decreasing intensity of the transmission peak near $700 \mathrm{~nm}$. Cycle 0 shows the spectrum measured prior to film deposition. Inset in Figure 3a shows dynamic response measured near $700 \mathrm{~nm}$ during deposition of TSPP layer.

Fig. 4. peak wavelength shift near $700 \mathrm{~nm}$ vs. the number of PAH/TSPP deposition cycles for TSPP outermost layers in air measured using tapered fibres with waist diameter of: squares, 9 $\mu \mathrm{m}$; circles, $10 \mu \mathrm{m}$; and triangles, $12 \mu \mathrm{m}$.

Fig. 5. (a) Transmission difference spectra obtained by subtracting a spectrum measured in the 
$100 \mathrm{ppm}$ ammonia atmosphere from the spectrum measured in air with the tapered fibres of 9 , 10 , and $12 \mu \mathrm{m}$ modified with a (PAH/TSPP $)_{5}$ film, (b) transmission difference spectra of the 10 $\mu \mathrm{m}$ tapered fibre measured at given ammonia concentrations from $10 \mathrm{ppm}$ to $100 \mathrm{ppm}$, (c) transmission spectra of the $9 \mu \mathrm{m}$ tapered fibre measured before and after 50 and $100 \mathrm{ppm}$ ammonia exposures, and (d) dynamic responses of the 10 and $12 \mu \mathrm{m}$ tapered fibres to the varying ammonia concentration (from $100 \mathrm{ppm}$ to $10 \mathrm{ppm}$ ) recorded at $706 \mathrm{~nm}$, where arrows indicate the admission time of ammonia and air into the measurement chamber. The inset of Fig. 5(d) shows a calibration curve plotted from the difference spectra data taken at $706 \mathrm{~nm}$ : squares and circles show the data of the 10 and $12 \mu \mathrm{m}$ tapered fibres, respectively.

Fig. 6. (a) Transmission spectra of the $10 \mu \mathrm{m}$ tapered fibre modified with PAH/TSPP film measured before and after change of the relative humidity ; (b) dynamic responses of the 10 $\mu \mathrm{m}$ tapered fibre to the varying $\mathrm{rH}$ from 70 to $10 \%$ and back recorded at $706 \mathrm{~nm}$, where lines indicate the admission time of dry air into the measurement chamber. 
Fig. 1

(a)

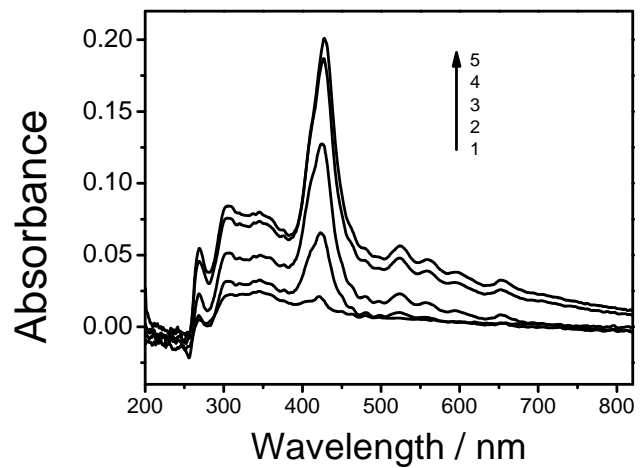

(b)

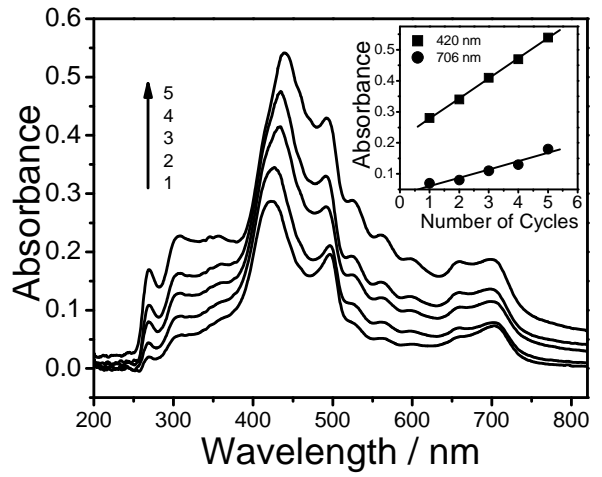

Fig. 1. Evolution of the absorption spectra during deposition of a 5-cycle PAH/TSPP alternate film onto a quartz substrate measured with an outermost layer of (a) PAH and (b) TSPP, respectively. Arrows indicate the number of bilayers deposited and the inset of Fig. 1b shows peak absorbance vs. the number of deposited layers measured near $420 \mathrm{~nm}$ (squares) and 706 $\mathrm{nm}$ (circles). 
Fig. 2

(a)
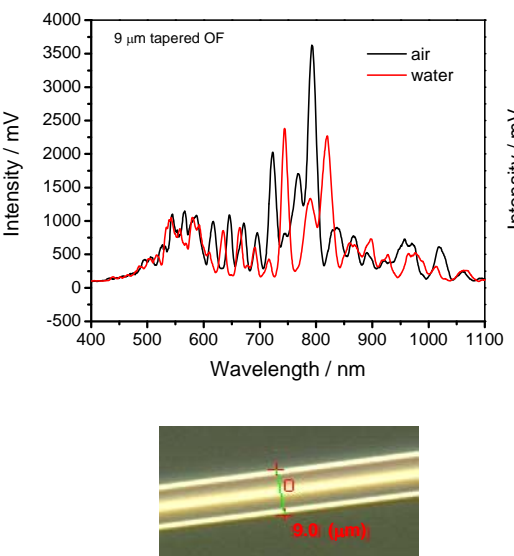

(b)
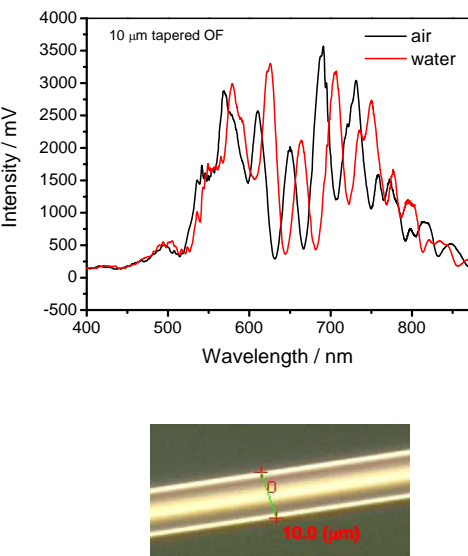

(c)
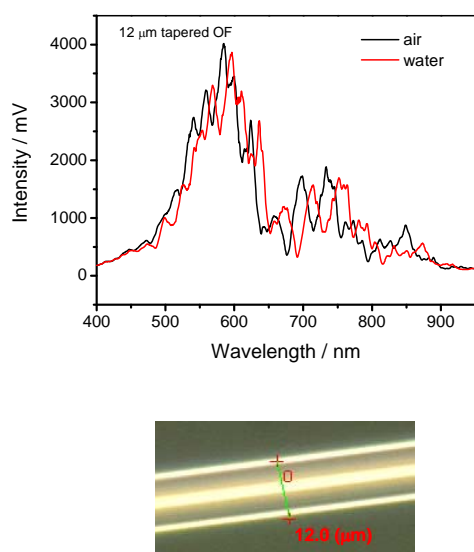

Fig. 2. Comparison of the transmission spectra measured in air and water using tapered optical fibres with a taper diameter of (a) $9 \mu \mathrm{m}$, (b) $10 \mu \mathrm{m}$, and (c) $12 \mu \mathrm{m}$. Optical images show the tapered region of the optical fibres with different waist diameters. 
Fig. 3

(a)

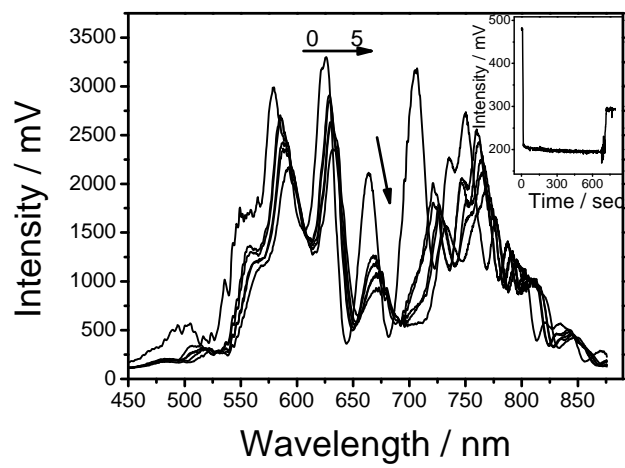

(c)

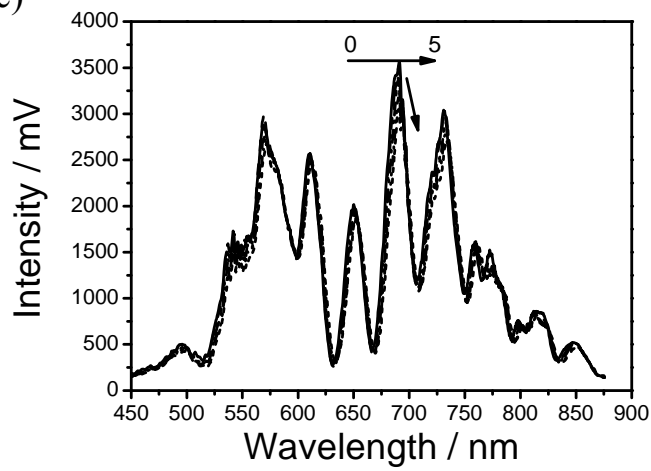

(b)

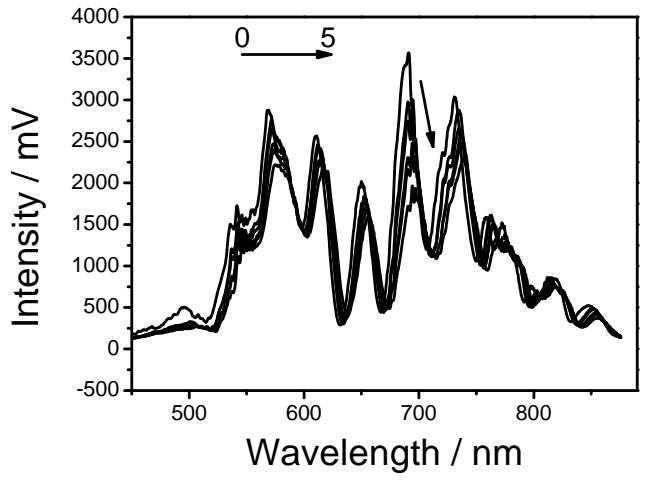

(d)

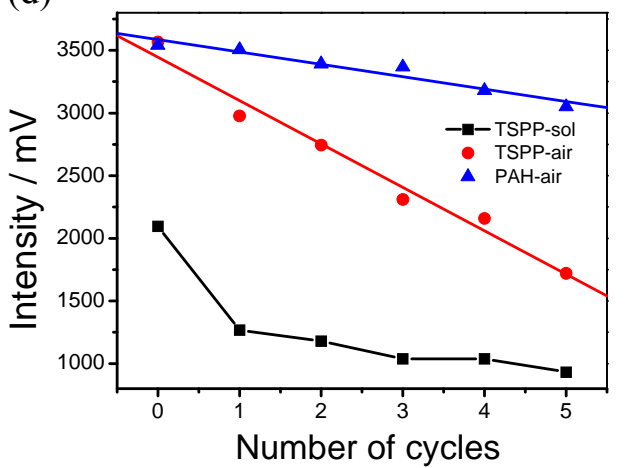

Fig. 3. Evolution of the transmission spectra of the $10 \mu \mathrm{m}$ tapered optical fibre during deposition of a five-cycle PAH/TSPP alternate film (a) Measured in TSPP solution and measured after drying the optical fibre with an outermost layer of (b) TSPP and (c) PAH. (d) Peak intensity measured near $700 \mathrm{~nm}$ plotted vs. the number of PAH/TSPP deposition cycles for TSPP and PAH outermost layers in water and air; Horizontal arrows indicate the number of layers deposited and the direction in which the transmission peaks are shifted. The downward arrows indicate the decreasing intensity of the transmission peak near $700 \mathrm{~nm}$. Cycle 0 shows the spectrum measured prior to film deposition. Inset in Figure 3a shows dynamic response measured near $700 \mathrm{~nm}$ during deposition of TSPP layer. 
Fig. 4

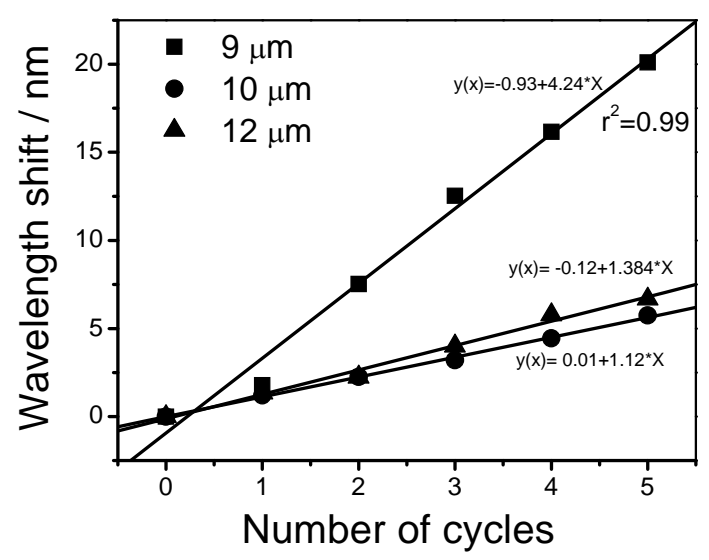

Fig. 4. Peak wavelength shift near $700 \mathrm{~nm}$ vs. the number of PAH/TSPP deposition cycles for TSPP outermost layers in air measured using tapered fibres with waist diameter of: squares, 9 $\mu \mathrm{m}$; circles, $10 \mu \mathrm{m}$; and triangles, $12 \mu \mathrm{m}$. 
Fig. 5

(a)

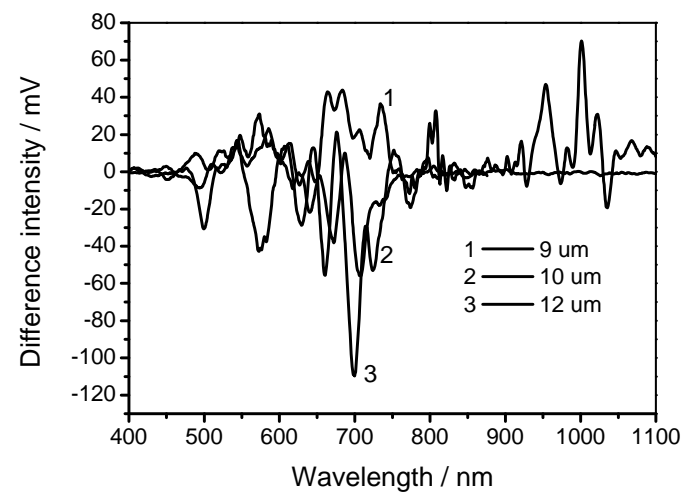

(c)

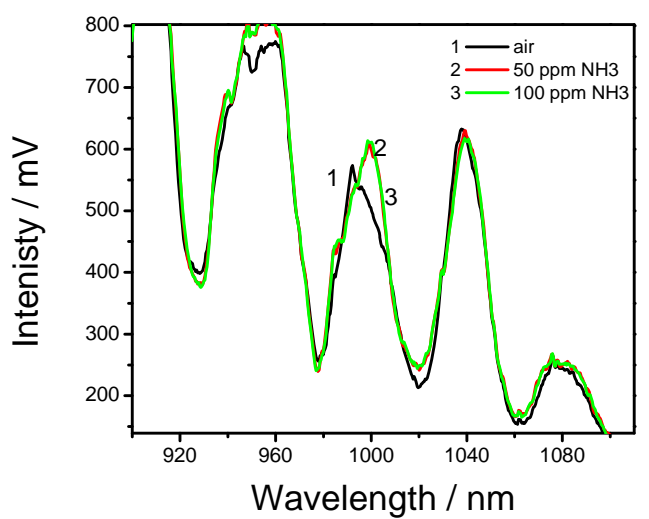

(b)

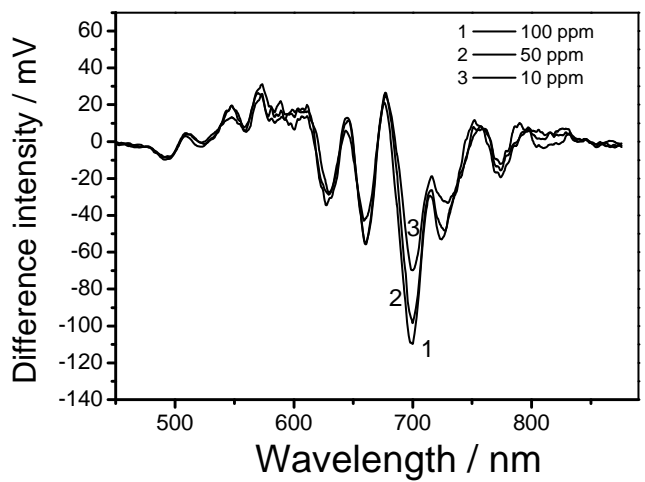

(d)

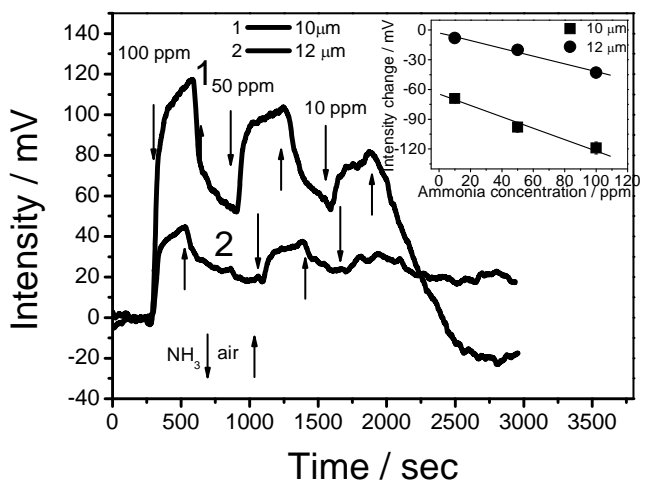

Fig. 5. (a) Transmission difference spectra obtained by subtracting a spectrum measured in the $100 \mathrm{ppm}$ ammonia atmosphere from the spectrum measured in air with the tapered fibres of 9 , 10 , and $12 \mu \mathrm{m}$ modified with a (PAH/TSPP $)_{5}$ film, (b) transmission difference spectra of the 10 $\mu \mathrm{m}$ tapered fibre measured at given ammonia concentrations from $10 \mathrm{ppm}$ to $100 \mathrm{ppm}$, (c) transmission spectra of the $9 \mu \mathrm{m}$ tapered fibre measured before and after 50 and $100 \mathrm{ppm}$ ammonia exposures, and (d) dynamic responses of the 10 and $12 \mu \mathrm{m}$ tapered fibres to the varying ammonia concentration (from $100 \mathrm{ppm}$ to $10 \mathrm{ppm}$ ) recorded at $706 \mathrm{~nm}$, where arrows indicate the admission time of ammonia and air into the measurement chamber. The inset of Fig. 5(d) shows a calibration curve plotted from the difference spectra data taken at $706 \mathrm{~nm}$ : squares and circles show the data of the 10 and $12 \mu \mathrm{m}$ tapered fibres, respectively. 
Fig. 6

(a)

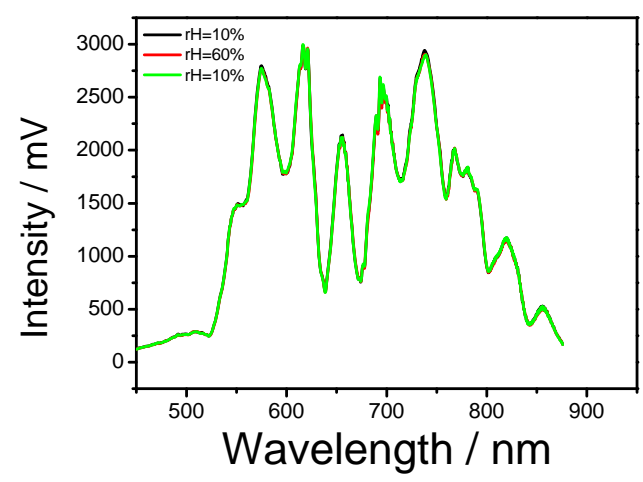

(b)

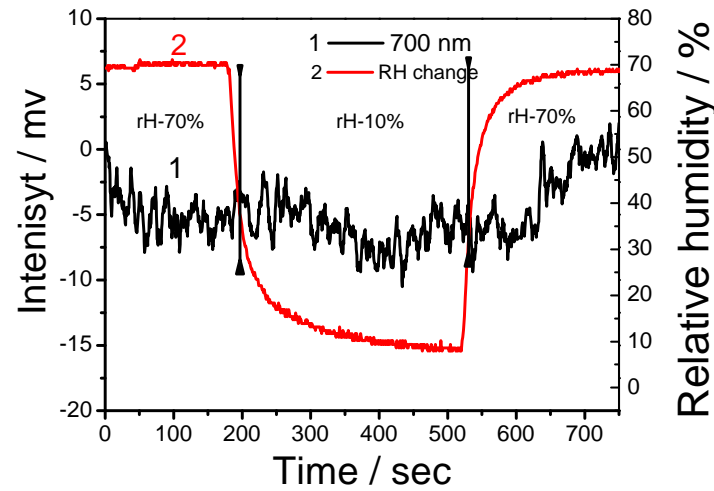

Fig. 6. (a) Transmission spectra of the $10 \mu \mathrm{m}$ tapered fibre modified with a 5-cycle PAH/TSPP film measured before and after change of the relative humidity and (b) dynamic responses of the $10 \mu \mathrm{m}$ tapered fibre to the varying $\mathrm{RH}$ from 70 to $10 \%$ and backwards recorded at $706 \mathrm{~nm}$, where lines indicate the admission time of dry air into the measurement chamber; line 1, sensor response; and line 2, RH change measured using humidity logger. 